FTUAM 93/27

NIKHEF-H 93/17

UPRF 93/376

\title{
Abelian Chern-Simons theory as the strong large-mass limit of topologically massive abelian gauge theory: the Wilson loop
}

\author{
G. Giavarini \\ INFN Gruppo collegato di Parma and Dipartimento \\ di Fisica dell'Università di Parma, \\ Viale delle Scienze, 43100 Parma, Italy \\ C. P. Martin \\ Departamento de Física Teórica, C-XI, Universidad Autónoma de Madrid, \\ Cantoblanco, 28049 Madrid, Spain \\ F. RuIz Ruiz \\ NIKHEF-H, Postbus 41882, 1009 DB Amsterdam, The Netherlands
}

\begin{abstract}
We show that the renormalized vacuum expectation value of the Wilson loop for topologically massive abelian gauge theory in $\mathbb{R}^{3}$ can be defined so that its largemass limit be the renormalized vacuum expectation value of the Wilson loop for abelian Chern-Simons theory also in $\mathbb{R}^{3}$.
\end{abstract}

(To appear in Nuclear Physics B) 


\section{Introduction}

Abelian Chern-Simons theory on an oriented three-dimensional riemannian manifold $\mathcal{M}$ is perhaps the simplest instance of a topological field theory. Its classical action $S_{A C S}$ is given by the metric-independent integral

$$
S_{A C S}=-\frac{i}{4 \theta} \int_{\mathcal{M}} A \wedge d A,
$$

where $A$ is a $U(1)$ gauge field on $\mathcal{M}$. The interest in this theory is that it provides field theoretical definitions of topological invariants of both the manifold and the curves lying on it. For example, if $\mathcal{M}$ is compact, it is well known that the partition function gives a topological invariant of $\mathcal{M}$ known as the Ray-Singer torsion [1]. It is also well understood that if $\mathcal{M}=\mathbb{R}^{3}$, the vacuum expectation value

$$
W_{A C S}(C)=\left\langle\exp \left(i \oint_{C} d x^{\mu} A_{\mu}\right)\right\rangle_{A C S}
$$

of the Wilson loop operator along a simple (i.e. without self-intersections) closed curve $C$ in $\mathcal{M}$ can be related to a topological invariant of the curve $C$ known as the self-linking number [2] [3]. Let us discuss this last point in somewhat more detail. Using that the action $S_{A C S}$ is quadratic in $A$, the path integral in eq. (1.2) can be explicity performed. In the covariant Landau gauge $\partial A=0$ one obtains

$$
W_{A C S}(C)=\exp \left\{-\frac{1}{2} \int_{0}^{\ell} d \tau_{1} \int_{0}^{\ell} d \tau_{2} \dot{x}^{\mu}\left(\tau_{1}\right)\left\langle A_{\mu}\left(x\left(\tau_{1}\right)\right) A_{\nu}\left(x\left(\tau_{2}\right)\right)\right\rangle_{A C S} \dot{x}^{\nu}\left(\tau_{2}\right)\right\}
$$

where $\ell$ and $\tau$ are respectively the length and the natural length parameter of the curve and

$$
\left\langle A_{\mu}(x) A_{\nu}(y)\right\rangle_{A C S}=\frac{i \theta}{2 \pi} \epsilon_{\mu \rho \nu} \frac{(x-y)^{\rho}}{|x-y|^{3}}
$$

is the gauge field propagator. Note however that the propagator $\left\langle A_{\mu}(x) A_{\nu}(y)\right\rangle_{A C S}$ is not well defined at $x=y$ so the right-hand side in eq. (1.3) becomes dependent 
on the regularization prescription used to regularize $\left\langle A_{\mu}(x) A_{\nu}(y)\right\rangle_{A C S}$ at $x=y^{\star}$. The point is that this prescription can be chosen so that $W_{A C S}(C)$ yields the Gauss self-linking number $\mathrm{SL}(C)$ of the curve $C$. Indeed, if a framing of the curve, defined by a unit vector field $n^{\mu}(\tau)$ orthogonal to $x^{\mu}(\tau)$, is used as a regulator, the exponent on the right-hand side in eq. (1.3) is equal to $i \theta$ times the curve's self-linking number [3]:

$$
\begin{aligned}
\lim _{\eta \rightarrow 0} & \left\{\frac{1}{2} \int_{0}^{\ell} d \tau_{1} \int_{0}^{\ell} d \tau_{2} \dot{x}^{\mu}\left(\tau_{1}\right)\left\langle A_{\mu}\left(x\left(\tau_{1}\right)\right) A_{\nu}\left(x\left(\tau_{2}\right)+\eta n\left(\tau_{2}\right)\right)\right\rangle_{A C S}\left(\dot{x}^{\nu}\left(\tau_{2}\right)+\eta \dot{n}^{\nu}\left(\tau_{2}\right)\right)\right\} \\
& =-i \theta \operatorname{SL}(C) .
\end{aligned}
$$

As a result, $W_{A C S}(C)$ becomes

$$
W_{A C S}^{r e n}(C)=\lim _{\eta \rightarrow 0} W_{A C S}^{r e g}(C)=e^{i \theta \operatorname{SL}(C)} .
$$

One can think of this equation as defining a renormalization scheme in which the renormalized vacuum expectation value of the Wilson loop along the curve $C$ gives the exponential of $i \theta$ times the self-linking number of the curve.

It is important to stress that the value of the integral on the right-hand side in eq. (1.3) depends on the regularization chosen to define $\dot{x}^{\mu}\left(\tau_{1}\right)\left\langle A_{\mu}\left(x\left(\tau_{1}\right)\right) A_{\nu}\left(x\left(\tau_{2}\right)\right)\right\rangle_{A C S} \dot{x}^{\nu}\left(\tau_{2}\right)$ at $x\left(\tau_{1}\right)=x\left(\tau_{2}\right)^{\dagger}$ and that not every choice of regularization and renormalization renders $W_{A C S}(C)$ a topological invariant. For instance, the regularization and

$\star$ Rigourously speaking, the propagator $\left\langle A_{\mu}(x) A_{\nu}(y)\right\rangle_{A C S}$ is a distribution of which it is known that on any open set in $\mathbb{R}^{3} \times \mathbb{R}^{3}-\{x=y\}$ is defined by the function on the righthand side in eq. (1.4). The problem is that the domain of integration in eq. (1.3) includes $x=y$ and so the value of the integral depends on the definition of $\left\langle A_{\mu}(x) A_{\nu}(y)\right\rangle_{A C S}$ as a distribution. But the process of extending a function to a distribution is in general not unique and is what in quantum field theory is called regularization. Think for example of the distributions principal value $\mathrm{PV}(1 / x)$ and finite part $\mathrm{FP}(1 / x)$ : they are both associatd to the function $1 / x$ but as distributions are different.

$\dagger$ Note in contrast that contributions to $W_{A C S}(C)$ from compact regions $\left|x\left(\tau_{1}\right)-x\left(\tau_{2}\right)\right| \geq \varepsilon>$ 0 are regularization independent since the absolute value of the propagator $\left\langle A\left(x\left(\tau_{1}\right)\right) A\left(x\left(\tau_{2}\right)\right)\right\rangle_{A C S}$ is bounded in these regions. 
renormalization used by Polyakov [4] gives for $W_{A C S}(C)$ the exponential of $i \theta$ times the writhing number $w(C)$ of the curve $C$, but is well known that the writhing number is not a topological invariant [3] [5]. Thus a definition of abelain ChernSimons theory by means of Polyakov's regularization, though useful for other purposes [4], lacks topological meaning. In the sequel, whenever we refer to quantum abelian Chern-Simons theory, we shall be referring to the topologically invariant formulation in eqs. (1.5) and (1.6).

Let us consider now a different gauge theory, namely topologically massive abelian gauge theory. Its classical action on the manifold $\mathcal{M}$ endowed with metric $g_{\mu \nu}$ has the form [6] [7]

$$
S_{T M A}=\int_{\mathcal{M}}\left(-\frac{i}{4 \theta} A \wedge d A+\frac{1}{16 m} F \wedge * F\right),
$$

where $m$ is a parameter with dimensions of mass. The action now depends on the metric through the Maxwell term $F \wedge * F$. However, for gauge connections that grow slower than $m^{1 / 2}$ the metric dependence disappears in the large $m$ limit. So starting from $S_{T M A}$ and sending $m$ to infinity one recovers the Chern-Simons action in eq. (1.1). We would like to know whether this classical convergence of topologically massive abelian gauge theory to abelian Chern-Simons theory as $m$ goes to infinity holds at the quantum level. More precisely, if we restrict ourselves to $\mathcal{M}=\mathbb{R}^{3}$, the question we want to address is: Is it possible to define the renormalized vacuum expectation value of the Wilson loop in topologically massive abelian gauge theory so that its large $m$ limit yields the topological invariant in eq. (1.6)? The purpose of this paper is to answer this question in the affirmative (for an analysis of the effective action in the non-abelian case, see ref. [7]).

The layout of the paper is as follows. In sect. 2 we introduce a regularization method and a renormalization scheme for the vacuum expectation value of the topologically massive Wilson loop that yields the topological invariant in eq. (1.6) as $m$ goes to infinity in a strong sense. Sect. 2 also contains our results and conclusions, leaving for sect. 3 the details of our computations. 


\section{Results and conclusions}

Our concern here is the topologically massive vacuum expectation value

$$
W_{T M A}(C)=\left\langle\exp \left(i \oint_{C} d x^{\mu} A_{\mu}\right)\right\rangle_{T M A}
$$

of the Wilson loop operator along a simple closed curve $C$ contained in $\mathbb{R}^{3}$. In the covariant Landau gauge, the latter is given by

$$
W_{T M A}(C)=\exp [-F(C, m)]
$$

where

$$
F(C, m)=\frac{1}{2} \int_{0}^{\ell} d \tau_{1} \int_{0}^{\ell} d \tau_{2} \dot{x}^{\mu}\left(\tau_{1}\right)\left\langle A_{\mu}\left(x\left(\tau_{1}\right)\right) A_{\nu}\left(x\left(\tau_{2}\right)\right)\right\rangle_{T M A} \dot{x}^{\nu}\left(\tau_{2}\right)
$$

and

$$
\left\langle A_{\mu}(x) A_{\nu}(y)\right\rangle_{T M A}=2 m \int \frac{d^{3} p}{(2 \pi)^{3}}\left\{\frac{m \epsilon_{\mu \rho \nu} p^{\rho}}{p^{2}\left(p^{2}+m^{2}\right)}+\frac{p^{2} g_{\mu \nu}-p_{\mu} p_{\nu}}{p^{2}\left(p^{2}+m^{2}\right)}\right\} e^{i p(x-y)}
$$

is the propagator of the gauge field. In deriving eqs. (2.1)-(2.3) we have rescaled $A \rightarrow A \theta$ and $m \rightarrow m \theta / 2$ in $S_{A C S}$ and $S_{T M A}$ so as to be rid of the parameter $\theta$ (note that this will not affect the analysis of the large $m$ limit since $\theta$ remains finite as $m$ goes to infinity). Now, on every compact domain $\{(x, y):|x-y| \geq \varepsilon>0\}$ the propagator $\left\langle A_{\mu}(x) A_{\nu}(y)\right\rangle_{T M A}$ is well defined and converges uniformly to $\left\langle A_{\mu}(x) A_{\nu}(y)\right\rangle_{A C S}$ as $m$ goes to infinity. However, when $x=y$, $\left\langle A_{\mu}(x) A_{\nu}(y)\right\rangle_{T M A}$ diverges linearly and the double integral $F(C, m)$ becomes logarithmically divergent [8]. So to have a sensible definition for $W_{T M A}(C)$ and to be able to later evaluate its large $m$ limit, it is necessary to provide $F(C, m)$ with 
a well defined meaning. This is achieved via renormalization and entitles regularization as a first step. Regularization can be accomplished by regularizing the integrand

$$
f\left(C, m ; \tau_{1}, \tau_{2}\right)=\dot{x}^{\mu}\left(\tau_{1}\right)\left\langle A_{\mu}\left(x\left(\tau_{1}\right)\right) A_{\nu}\left(x\left(\tau_{2}\right)\right)\right\rangle_{T M A} \dot{x}^{\nu}\left(\tau_{2}\right)
$$

in eq. (2.2) in such a way that (i) one recovers the finite unregularized value of $f\left(C, m ; \tau_{1}, \tau_{2}\right)$ for $x\left(\tau_{1}\right) \neq x\left(\tau_{2}\right)$ when the regulator is removed, and (ii) no singularity occurs for $x\left(\tau_{1}\right)=x\left(\tau_{2}\right)$ when the regulator is on.

Refs. [8] propose to use a framing of the curve, defined by a unit vector field $n^{\mu}(\tau)$ normal to $x^{\mu}(\tau)$, as a regulator. Furthermore, they show that

$$
\begin{aligned}
\lim _{m \rightarrow \infty} \lim _{\eta \rightarrow 0} \operatorname{Im} & \left\{\frac{1}{2} \int_{0}^{\ell} d \tau_{1} \int_{0}^{\ell} d \tau_{2} \dot{x}^{\mu}\left(\tau_{1}\right)\left\langle A_{\mu}\left(x\left(\tau_{1}\right)\right) A_{\nu}\left(x\left(\tau_{2}\right)+\eta n\left(\tau_{2}\right)\right)\right\rangle_{T M A}\left(\dot{x}^{\nu}\left(\tau_{2}\right)+\eta \dot{n}^{\nu}\left(\tau_{2}\right)\right)\right\} \\
& =-w(C)
\end{aligned}
$$

Let us stop for a moment and understand this result. Taking $x \neq y$, computing the Fourier transform in eq. (2.3) and retaining only the imaginary part, one obtains $[8]$

$\Delta_{\mu \nu}(x-y) \equiv \operatorname{Im}\left[\left\langle A_{\mu}(x) A_{\nu}(y)\right\rangle_{T M A}\right]=\frac{i}{2 \pi} \epsilon_{\mu \rho \nu} \frac{(x-y)^{\rho}}{|x-y|^{3}}\left[1-(1+m|x-y|) e^{-m|x-y|}\right]$

Using that $\Delta_{\mu \nu}(x-y)$ remains bounded as $x \rightarrow y$, one concludes that the limit $\eta \rightarrow 0$ in eq. (2.5) is independent of $n^{\mu}(\tau)$ and equal to

$$
\frac{1}{2} \int_{0}^{\ell} d \tau_{1} \int_{0}^{\ell} d \tau_{2} \dot{x}^{\mu}\left(\tau_{1}\right) \Delta_{\mu \nu}\left(x\left(\tau_{1}\right)-x\left(\tau_{2}\right)\right) \dot{x}^{\nu}\left(\tau_{2}\right) .
$$

It is important to bear in mind that this way to proceed assigns the propagator an imaginary part at $x=y$ by first taking $x \neq y$ and then sending $x \rightarrow y$ in 
the result but that this does not mean that the imaginary part of the propagator is unambiguously defined at $x=y$, for as already discussed the propagator itself is undetermined at $x=y$. Finally, one shows [8] that the large $m$ limit of eq. (2.6) is equal to minus the writhing number $w(C)$. Now, since $(2.6)$ is equal to the "naive imaginary part" of $F(C, m)$ in eqs. (2.1)-(2.2), one would conclude that the large $m$ limit of $W_{T M A}(C)$ yields Polyakov's phase factor $e^{i w(C)}$. Consequently, abelian Chern-Simons theory could not be obtained as the strong large $m$ limit of topologically massive abelian gauge theory. This is the conclusion reached in refs. $[8]$.

The previous analysis is however incomplete, for it overlooks that the double integral $F(C, m)$ is logarithmically divergent and that therefore it does not make sense to talk about its imaginary part as being finite and equal to (2.6), as done in refs. [8]. The divergence of $F(C, m)$ will show up as a pole in dimensional regularization, the regularization method we will be using here. To make rigorous statements about the large $m$ behaviour of $W_{T M A}(C)$, one first has to renormalize it. This is tantamount to renormalizing the double integral $F(C, m)$. Once $F(C, m)$ has been renormalized, it will then make sense to compute its imaginary part and ask whether or not it leads to Polyakov's phase factor $e^{i w(C)}$ when $m$ is sent to infinity. Of course, if the imaginary part of the renormalized $F(C, m)$ did not depend on the renormalization prescription, the large $m$ limit of $W_{T M A}(C)$ would yield Polyakov's phase factor. Were this the case, the claim made in refs. [8] would be correct and abelian Chern-Simons theory could not be obtained from topologically massive abelian gauge theory by taking the large $m$ limit in a strong sense. In this paper, we shall see however that the imaginary part of the renormalized double integral $F(C, m)$ does depend on the renormalization prescription and, moreover, that there is a definition of the renormalized value of $W_{T M A}(C)$ that converges to $W_{A C S}^{r e n}(C)$ in eq. (1.6) as $m$ goes to infinity.

To renormalize the vacuum expectation value of the topologically massive Wilson loop, we first introduce a regularization method and then provide a renormalization scheme. Our regularization method combines a framing of the curve and 
dimensional regularization in the following way. It defines $W_{T M A}^{r e g}(C)$ as

$$
W_{T M A}^{r e g}(C)=\exp \left[-F_{r e g}(C, m)\right]
$$

where

$$
F_{r e g}(C, m)=\int_{0}^{\ell} d \tau_{1} \int_{0}^{\ell} d \tau_{2} f_{r e g}\left(C, m ; \tau_{1}, \tau_{2}\right)
$$

and

$$
f_{\text {reg }}\left(C, m ; \tau_{1}, \tau_{2}\right)=f_{1}\left(\tau_{1}, \tau_{2}\right)+f_{2}\left(\tau_{1}, \tau_{2}\right)+f_{3}\left(\tau_{1}, \tau_{2}\right)
$$

The functions $f_{1,2,3}\left(\tau_{1}, \tau_{2}\right)$ are defined by the equations

$$
\begin{gathered}
f_{1}\left(\tau_{1}, \tau_{2}\right)=\dot{x}^{\mu}\left(\tau_{1}\right)\left\{\kappa^{3-D} \int \frac{d^{D} p}{(2 \pi)^{D}} \frac{\epsilon_{\mu \rho \nu} p^{\rho}}{p^{2}} e^{i p\left(x\left(\tau_{1}\right)-x\left(\tau_{2}\right)-\eta n\left(\tau_{2}\right)\right)}\right\}\left(\dot{x}^{\nu}\left(\tau_{2}\right)+\eta \dot{n}^{\nu}\left(\tau_{2}\right)\right) \\
f_{2}\left(\tau_{1}, \tau_{2}\right)=-\dot{x}^{\mu}\left(\tau_{1}\right)\left\{\kappa^{3-D} \int \frac{d^{D} p}{(2 \pi)^{D}} \frac{\epsilon_{\mu \rho \nu} p^{\rho}}{p^{2}+m^{2}} e^{i p\left(x\left(\tau_{1}\right)-x\left(\tau_{2}\right)\right)}\right\} \dot{x}^{\nu}\left(\tau_{2}\right) \\
f_{3}\left(\tau_{1}, \tau_{2}\right)=\dot{x}^{\mu}\left(\tau_{1}\right)\left\{m \kappa^{3-D} \int \frac{d^{D} p}{(2 \pi)^{D}} \frac{p^{2} g_{\mu \nu}-p_{\mu} p_{\nu}}{p^{2}\left(p^{2}+m^{2}\right)} e^{i p\left(x\left(\tau_{1}\right)-x\left(\tau_{2}\right)\right)}\right\} \dot{x}^{\nu}\left(\tau_{2}\right),
\end{gathered}
$$

where $n^{\mu}(\tau)$ denotes the unitary vector field orthogonal to the curve $C$ defining the framing and $\kappa$ the mass scale introduced by dimensional regularization. Note that our regularization depends on two regulators. One is $\eta$, the regulator governing the framing or point-splitting in eq. (2.10); the other one is $\varepsilon=D-3$, the dimensional regulator. The regulators $\eta$ and $\varepsilon$ are not to be removed in an arbitrary way when UV divergences arise: the prescription is to take first the limit $\varepsilon \rightarrow 0$ and then send $\eta$ to zero. We shall see that this, together with a suitable renormalization scheme, gives for the large $m$ limit of the Wilson loop the topological invariant in eq. (1.6). 
The reasons why eqs. (2.8)-(2.12) define a suitable regularization of the double integral $F(C, m)$ can be explained as follows. First, because $F_{\text {reg }}(C, m)$ defined in eq. (2.8) above is finite for $\eta \neq 0$ and $\varepsilon$ in a suitable domain in the complex plane. And secondly, because if

$$
U=[0, \ell] \times[0, \ell]-\left\{\left(\tau_{1}, \tau_{2}\right): \tau_{1}=\tau_{2}\right\}-\{(0, \ell)\}-\{(\ell, 0)\}
$$

denotes the subdomain for which $x\left(\tau_{1}\right)$ is never equal to $x\left(\tau_{2}\right)$ and $U^{c}$ is any simply connected and closed subset of $U$, it is not difficult to see that the following equations hold:

$$
\begin{aligned}
& \lim _{\eta \rightarrow 0} \lim _{D \rightarrow 3} f_{r e g}\left(C, m ; \tau_{1}, \tau_{2}\right)=f\left(C, m ; \tau_{1}, \tau_{2}\right) \quad \text { for all }\left(\tau_{1}, \tau_{2}\right) \text { in } U^{c} \\
& \lim _{\eta \rightarrow 0} \lim _{D \rightarrow 3} \iint_{U^{c}} d \tau_{1} d \tau_{2} f_{r e g}\left(C, m ; \tau_{1}, \tau_{2}\right)=\iint_{U^{c}} d \tau_{1} d \tau_{2} f\left(C, m ; \tau_{1}, \tau_{2}\right) .
\end{aligned}
$$

In other words, our prescription (i) provides a finite $F_{r e g}(C, m)$ and (ii) does not change the value of unregularized contributions to $F(C, m)$ as far as the latter are well defined. So in accordance with the principles of renormalization theory [9] it defines a regularization method.

The regularized vacuum expectation value of the topologically massive Wilson loop as computed with this regularization method turns out to be (see sect. 3 for its derivation)

$$
W_{T M A}^{r e g}(C)=\exp \left\{i \operatorname{SL}(C)-\frac{m}{2 \pi}\left[\frac{1}{D-3}+\frac{1}{2} \ln \left(\frac{m^{2}}{4 \pi \kappa^{2}}\right)+\frac{1}{2} \gamma\right] \ell+\text { v.t. }\right\}
$$

where $\operatorname{SL}(C)$ is the self-linking number of the curve $C, \gamma$ is the Euler constant and "v.t." stands for contributions that vanish as $D \rightarrow 3, \eta \rightarrow 0$ and $m \rightarrow \infty$. As anounced earlier, $W_{T M A}^{r e g}(C)$ becomes singular as $D$ goes to 3 . To remove this 
divergence, we follow refs. [10] and define the renormalized vacuum expectation value of the topologically massive abelian Wilson loop as

$$
W_{T M A}^{r e n}(C)=\lim _{\eta \rightarrow 0} \lim _{D \rightarrow 3} e^{M \ell} W_{T M A}^{r e g}(C)
$$

where

$$
M=\frac{m}{2 \pi}\left[\frac{1}{D-3}+\frac{1}{2} \ln \left(\frac{m^{2}}{4 \pi \kappa^{2}}\right)+\frac{1}{2} \gamma\right] .
$$

As usual [10], $M$ can be physically interpreted as the renormalization constant for the mass of a test particle being driven along the the curve $C$. From eqs. (2.13) and (2.14) we conclude that

$$
W_{T M A}^{r e n}(C)=e^{i \mathrm{SL}(C)+v(m)}
$$

with $v(m)$ collecting the contributions in "v.t." that do not vanish as $D \rightarrow 3$ and $\eta \rightarrow 0$ but do vanish as $m \rightarrow \infty$,

$$
\lim _{m \rightarrow \infty} v(m)=0
$$

Eq. (2.14) defines our renormalization scheme and eq. (2.15) displays the renormalized vacuum expectation value $W_{T M A}^{r e n}(C)$ in this scheme of the topologically massive Wilson loop. Since $W_{T M A}^{r e n}(C)$ is finite, we are now in a position to study its large $m$ limit properly. The latter is trivial, for eqs. (2.15) and (2.16) imply

$$
\lim _{m \rightarrow \infty} W_{T M A}^{r e n}(C)=e^{i \mathrm{SL}(C)},
$$

that together with eq. (1.6) lead us to the main conclusion of this paper: Abelian Chern-Simons theory can be understood as the strong large $m$ limit of topologically massive abelian gauge theory. This conclusion agrees with the results obtained within the hamiltonian formalism [11] and entails the definition of the renormalized topologically massive Wilson loop as provided by eqs. (2.13) and (2.14). Some final comments are in order: 
Comment 1. It is not difficult to convince oneself that if one computes the regularized vacuum expectation value of the topologically massive Wilson loop using the pure framing or point-splitting regularization advocated in refs. [8], one obtains

$$
W_{T M A}^{\prime r e n}(C)=\lim _{\eta \rightarrow 0} e^{M^{\prime} \ell} W_{T M A}^{\prime r e g}(C)=e^{i w(C)+v(m)}
$$

for an appropriate choice of $M^{\prime}$. Here $w(C)$ denotes as usual the writhing number of the curve $C$. It then follows that

$$
\lim _{m \rightarrow \infty} W_{T M A}^{\prime r e n}=e^{i w(C)}
$$

Eqs. (2.18) and (2.19) tell us the way the results presented in refs. [8] should be understood, namely as corresponding to a particular renormalization scheme defined from a particular regularization method. The conclusion in refs. [8] should therefore be restated to read that for the particular choice of renormalization scheme (2.18) the large $m$ limit of the renormalized Wilson loop is not a topological invariant. Our renormalization scheme though yields a topologically invariant large $m$ limit.

Comment 2. According to general principles of renormalized quantum field theory [10] [12], since $W_{T M A}^{r e n}(C)$ and $W_{T M A}^{\prime r e n}(C)$ in eqs. (2.15) and (2.18) correspond to two different renormalization schemes, there should be a local parametrizationinvariant counterterm that transforms $W_{T M A}^{r e n}(C)$ into $W_{T M A}^{\prime r e n}(C)$. Such a counterterm does indeed exist and has the form

$$
e^{i \mathrm{TW}(C)},
$$

where $\operatorname{TW}(C)$ is the functional

$$
\mathrm{TW}(C)=\frac{1}{2 \pi} \int_{0}^{\ell} d \tau \epsilon_{\mu \rho \nu} \dot{n}^{\nu}(\tau) \dot{x}^{\rho}(\tau) n^{\nu}(\tau)
$$

To see the latter, it is enough to multiply $W_{T M A}^{\prime r e n}(C)$ by $e^{i \mathrm{TW}(C)}$ and use the 
identity $\mathrm{SL}(C)=w(C)+\mathrm{TW}(C)[5]$ since then

$$
W_{T M A}^{r e n}(C)=e^{i \mathrm{TW}(C)} W_{T M A}^{\prime r e n}(C)
$$

The number $\mathrm{TW}(C)$ is the twist of the ribbon determined by the curve $C$ and its framing $n^{\mu}(\tau)$ and is not a topological invariant [5]. Notice that $e^{i \operatorname{TW}(C)}$ is a perfectly allowed counterterm since $\mathrm{TW}(C)$ is a local integrated functional of $x^{\nu}(\tau), n^{\nu}(\tau)$ and its derivatives and is parametrization invariant. Note also that TW $(C)$ has support on the compact segment line $\left\{\left(\tau_{1}, \tau_{2}\right): \tau_{1}=\tau_{2}\right\}$ only. This can be easily understood if one takes into account that contributions to $F(C, m)$ from non-coincident points $x\left(\tau_{1}\right) \neq x\left(\tau_{2}\right)$ are regularization independent and do not enter in neither of the renormalizations performed in eqs. (2.14) and (2.18), so any difference between $W_{T M A}^{r e n}(C)$ and $W_{T M A}^{\text {ren }}(C)$ has to be of the form the exponential of the integral of a function with support only on the compact segment line $\left\{\left(\tau_{1}, \tau_{2}\right): \tau_{1}=\tau_{2}\right\}$. To summarize, once a topologically massive renormalized Wilson loop has been obtained along the lines discussed in refs. [8], a finite renormalization (or change of renormalization scheme) by means of the counterterm $e^{i \mathrm{TW}(C)}$ leads to a renormalized Wilson loop whose large $m$ limit is a topological invariant. This is the same topological invariant that our renormalization scheme yields automatically.

\section{Computations}

In this section we give some details of the derivation of the regularized vacuum expectation value of the topologically massive Wilson loop in eq. (2.13). We shall prove the three following partial results:

$$
F_{1}(C) \equiv \int_{0}^{\ell} d \tau_{1} \int_{0}^{\ell} d \tau_{2} f_{1}\left(\tau_{1}, \tau_{2}\right)=-i \mathrm{SL}(C)+\text { v.t. }
$$




$$
F_{2}(C, m) \equiv \int_{0}^{\ell} d \tau_{1} \int_{0}^{\ell} d \tau_{2} f_{2}\left(\tau_{1}, \tau_{2}\right)=0+\text { v.t. }
$$

and

$F_{3}(C, m) \equiv \int_{0}^{\ell} d \tau_{1} \int_{0}^{\ell} d \tau_{2} f_{3}\left(\tau_{1}, \tau_{2}\right)=\frac{m}{2 \pi}\left[\frac{1}{D-3}+\frac{1}{2} \ln \left(\frac{m^{2}}{4 \pi \kappa^{2}}\right)+\frac{1}{2} \gamma\right] \ell+$ v.t.

where "v.t." stands for contributions that vanish when the limits $D \rightarrow 3, \eta \rightarrow 0$ and $m \rightarrow \infty$ are taken in this order. The three equations (3.1)-(3.3), together with

$$
F_{r e g}(C, m)=F_{1}(C)+F_{2}(C, m)+F_{3}(C, m)
$$

and eq. (2.7), imply eq. (2.13). So to obtain eq. (2.13) all we have to do is derive eqs. (3.1)-(3.3).

$\underline{\text { Derivation of eq. }(3.1)}$

To perform the integral over the momenta in $f_{1}\left(\tau_{1}, \tau_{2}\right)$ in eq. $(2.10)$ we use

$$
\int \frac{d^{D} p}{(2 \pi)^{D}} \frac{e^{i p x}}{p^{2}}=\frac{\Gamma\left(\frac{D}{2}-1\right)}{4 \pi^{D / 2}} \frac{1}{|x|^{D-2}}
$$

This gives

$$
\begin{aligned}
F_{1}(C) & =\frac{i \kappa^{3-D} \Gamma(D / 2)}{4 \pi^{D / 2}} \\
& \times \int_{0}^{\ell} d \tau_{1} \int_{0}^{\ell} d \tau_{2} \epsilon_{\mu \rho \nu} \dot{x}^{\mu}\left(\tau_{1}\right) \frac{x^{\rho}\left(\tau_{1}\right)-x^{\rho}\left(\tau_{2}\right)-\eta n^{\rho}\left(\tau_{2}\right)}{\left|x\left(\tau_{1}\right)-x\left(\tau_{2}\right)-\eta n\left(\tau_{2}\right)\right|^{D}}\left(\dot{x}^{\nu}\left(\tau_{2}\right)+\eta \dot{n}^{\nu}\left(\tau_{2}\right)\right) .
\end{aligned}
$$

Noting now that the integral on the right-hand side is absolutely convergent at $D=3$, we have that the following equation holds in dimensional regularization 
[13]:

$\lim _{D \rightarrow 3} F_{1}(C)=\frac{i}{4 \pi} \int_{0}^{\ell} d \tau_{1} \int_{0}^{\ell} d \tau_{2} \epsilon_{\mu \rho \nu} \dot{x}^{\mu}\left(\tau_{1}\right) \frac{x^{\rho}\left(\tau_{1}\right)-x^{\rho}\left(\tau_{2}\right)-\eta n^{\rho}\left(\tau_{2}\right)}{\left|x\left(\tau_{1}\right)-x\left(\tau_{2}\right)-\eta n\left(\tau_{2}\right)\right|^{3}}\left(\dot{x}^{\nu}\left(\tau_{2}\right)+\eta \dot{n}^{\nu}\left(\tau_{2}\right)\right)$

Taking finally the limit $\eta \rightarrow 0$ and recalling eqs. (1.4) and (1.5), we conclude

$$
\lim _{\eta \rightarrow 0} \lim _{D \rightarrow 3} F_{1}(C)=-i \mathrm{SL}(C)
$$

$\underline{\text { Derivation of eq. }(3.2)}$

In the remainder of this section we show that eqs. (3.2) and (3.3) hold true for any analytic curve $x^{\mu}(\tau)$. As will become clear in a moment, analiticity is a technical requirement that will render uniformly convergent the series expansions we perform below. The convergence being uniform will make possible to integrate these series and to evaluate their large $m$ limit term by term. Our computations will then be strictly valid for analytic curves only, though we believe our results are correct for curves of class $C^{1}$. In this case however some other method should be employed to prove them.

Calling $R(\tau)$ to the radius of convergence of $x^{\mu}(\tau)$ and using that by assumption $R(\tau)$ is positive for all $\tau$, we can define $\delta$ such that

$$
0<\delta<\min \{R(\tau): 0 \leq \tau \leq \ell\}
$$

So writing $F_{2}(C, m)$ as

$$
F_{2}(C, m)=2 \int_{0}^{\ell} d \tau_{1} \int_{0}^{\tau_{1}} d \tau_{2} f_{2}\left(\tau_{1}, \tau_{2}\right)
$$

and splitting its domain of integration

$$
\mathcal{D}=\left\{\left(\tau_{1}, \tau_{2}\right): 0 \leq \tau_{1} \leq \ell, \quad 0 \leq \tau_{2} \leq \tau_{1}\right\}
$$


into three subdomains

$$
\begin{aligned}
& \mathcal{D}_{1}=\left\{\left(\tau_{1}, \tau_{2}\right): 0 \leq \tau_{1} \leq \ell, 0 \leq \tau_{1}-\tau_{2} \leq \delta\right\} \\
& \mathcal{D}_{2}=\left\{\left(\tau_{1}, \tau_{2}\right): \ell-\delta \leq \tau_{1} \leq \ell, \quad 0 \leq \tau_{2} \leq \tau_{1}-\ell+\delta\right\} \\
& \mathcal{D}_{3}=\mathcal{D}-\mathcal{D}_{1}-\mathcal{D}_{2}
\end{aligned}
$$

we have

$$
F_{2}(C, m)=\sum_{i=1}^{3} F_{2, \mathcal{D}_{i}}
$$

where

$$
F_{2, \mathcal{D}_{i}}=2 \iint_{\mathcal{D}_{i}} d \tau_{1} d \tau_{2} f_{2}\left(\tau_{1}, \tau_{2}\right)
$$

Using

$$
\int \frac{d^{D} p}{(2 \pi)^{D}} \frac{e^{i p x}}{p^{2}+m^{2}}=\frac{1}{(4 \pi)^{D / 2}} \int_{0}^{\infty} \frac{d \lambda}{\lambda^{D / 2}} e^{-\lambda m^{2}} e^{-x^{2} / 4 \lambda}
$$

for the integration over the momenta hidden in $f_{2}\left(\tau_{1}, \tau_{2}\right)$, we obtain

$$
\begin{gathered}
F_{2, \mathcal{D}_{i}}=-\frac{i \kappa^{3-D}}{(4 \pi)^{D / 2}} \iint_{\mathcal{D}_{i}} d \tau_{1} d \tau_{2} \int_{0}^{\infty} \frac{d \lambda}{\lambda^{(D+2) / 2}} \epsilon_{\mu \rho \nu} \dot{x}^{\mu}\left(\tau_{1}\right)\left(x^{\rho}\left(\tau_{1}\right)-x^{\rho}\left(\tau_{2}\right)\right) \dot{x}^{\nu}\left(\tau_{2}\right) \\
\times e^{-\lambda m^{2}} e^{-\left(x\left(\tau_{1}\right)-x\left(\tau_{2}\right)\right)^{2} / 4 \lambda} .
\end{gathered}
$$

In the sequel we consider $F_{2, \mathcal{D}_{i}}$ for each $i$ separately and show that

$$
\lim _{m \rightarrow \infty} \lim _{D \rightarrow 3} F_{2, \mathcal{D}_{i}}=0 \quad i=1,2,3
$$

This result, along with eq. (3.6), implies eq. (3.2). 
We start proving eq. (3.9) for $i=1$. Performing a change of variables $\left(\tau_{1}, \tau_{2}\right) \rightarrow\left(\tau_{1}, t\right)$, with $t=\tau_{1}-\tau_{2}$, the domain of integration becomes

$$
\mathcal{D}_{1}=\left\{\left(\tau_{1}, t\right): 0 \leq \tau_{1} \leq \ell, \quad 0 \leq t \leq \delta\right\}
$$

Since $x^{\mu}(\tau)$ is analytic with radius of convergence $R(\tau)$ larger than $\delta$ for all $\tau$, $x^{\mu}\left(\tau_{2}\right)$ admits a uniformly convergent power series on $\mathcal{D}_{1}$ :

$$
x^{\mu}\left(\tau_{2}\right)=x^{\mu}\left(\tau_{1}-t\right)=\sum_{n=0}^{\infty}\left[\frac{d^{n}}{d \tau_{1}^{n}} x^{\mu}\left(\tau_{1}\right)\right] \frac{(-t)^{n}}{n !} .
$$

Substituting eq. (3.10) in eq. (3.8) and using that uniform convergence implies that integration over $t$ can be performed term by term, we have

$$
F_{2, \mathcal{D}_{1}}=-\frac{i \kappa^{3-D}}{(4 \pi)^{D / 2}} \int_{0}^{\ell} d \tau_{1} \sum_{j=0}^{\infty} I_{j}\left(D, m, \tau_{1}, \delta\right)
$$

where $I_{j}\left(D, m, \tau_{1}, \delta\right)$ is given by

$$
I_{j}\left(D, m, \tau_{1}, \delta\right)=\sum_{k=0}^{[j / 4]} c_{j k}\left(\tau_{1}\right) \int_{0}^{\delta} d t t^{4+j} \int_{0}^{\infty} \frac{d \lambda}{\lambda^{(D+2+2 k) / 2}} e^{-\lambda m^{2}} e^{-t^{2} / 4 \lambda}
$$

Here $[x]$ denotes the integer part of $x$ and $c_{j k}\left(\tau_{1}\right)$ are functions of $x^{\mu}\left(\tau_{1}\right)$ and its derivatives that do not depend on $m$ nor $D$ and whose explicit form is not important. In obtaining eqs. (3.11) and (3.12) we have used that, being $\tau$ the natural length parameter, $|\dot{x}(\tau)|=1$ and $\dot{x}^{\mu}(\tau) \ddot{x}_{\mu}(\tau)=0$. Integrating over $\lambda$ in eq. (3.12) we obtain

$$
I_{j}\left(D, m, \tau_{1}, \delta\right)=2 \sum_{k=0}^{[j / 4]} c_{j k}\left(\tau_{1}\right)(2 m)^{\frac{D}{2}+k} \int_{0}^{\delta} d t t^{4+j-k-\frac{D}{2}} K_{\frac{D}{2}+k}(m t),
$$

with $K_{\alpha}(z)$ the modified Bessel function of third type and order $\alpha$. Using that 
for half-integer order $K_{\alpha}(z)$ takes the simple form

$$
K_{n+\frac{1}{2}}(z)=\sqrt{\frac{\pi}{2 z}} e^{-z} \sum_{p=0}^{n} \frac{(n+p) !}{p !(n-p) !} \frac{1}{(2 z)^{p}} \quad n=0,1,2, \ldots
$$

it is straightforward to see that the integral over $t$ in eq. (3.13) is absolutely convergent at $D=3$ for all $m$ (convergence at $t=0$ does not pose any problem). This implies that the limit $D \rightarrow 3$ of $I_{j}\left(D, m, \tau_{1}, \delta\right)$ can be calculated by taking $D=3$ inside the integral in eq. (3.13). Thus, after a change of variables $u=m t$, we have

$$
\begin{aligned}
\lim _{D \rightarrow 3} I_{j}\left(D, m, \tau_{1}, \delta\right) & =4 \sqrt{\pi} \sum_{k=0}^{j} \frac{2^{k} c_{j k}\left(\tau_{1}\right)}{m^{2+j-2 k}} \\
& \times \sum_{p=0}^{k+1} \frac{(k+1+p) !}{2^{p} p !(k+1-p) !} \int_{0}^{m \delta} d u u^{2+j-k-p} e^{-u}
\end{aligned}
$$

The right-hand side obviously goes to zero as $m$ goes to infinity, hence

$$
\lim _{m \rightarrow \infty} \lim _{D \rightarrow 3} I_{j}\left(D, m, \tau_{1}, \delta\right)=0
$$

Together with uniform convergence of the series in eq. (3.11) this implies

$$
\lim _{m \rightarrow \infty} \lim _{D \rightarrow 3} F_{2, \mathcal{D}_{1}}=0
$$

in accordance with eq. (3.9). Using similar methods it is not difficult to prove that eq. (3.9) holds for $i=2$. Lastly we consider $i=3$. Noting that for all $\left(\tau_{1}, \tau_{2}\right)$ in $\mathcal{D}_{3}$ there exists a real number $r_{0}>0$ such that $\left|x\left(\tau_{1}\right)-x\left(\tau_{2}\right)\right| \geq r_{0}$, one may readily see that

$$
0 \leq\left|F_{2, \mathcal{D}_{3}}\right| \leq A_{0}(\delta) \kappa^{3-D}\left(\frac{m}{2 \pi r_{0}}\right)^{D / 2} K_{\frac{D}{2}}\left(m r_{0}\right)
$$

with $A_{0}(\delta)$ a positive constant that does not depend on $m$ nor $D$. So after taking 
the limits $D \rightarrow 3$ and $m \rightarrow \infty$ and using eq. (3.14), we conclude

$$
\lim _{m \rightarrow \infty} \lim _{D \rightarrow 3} F_{2, \mathcal{D}_{3}}=0
$$

This completes the proof of eq. (3.9), hence of eq. (3.2).

$\underline{\text { Derivation of eq. }(3.3)}$

To prove eq. (3.3) we proceed as follows. First we realize that the contribution to $F_{3}(C, m)$ of the term $p_{\mu} p_{\nu}$ in $f_{3}\left(\tau_{1}, \tau_{2}\right)$ is the integral of a total derivative along a closed curve, so it vanishes. This leaves us with

$$
F_{3}(C, m)=\frac{m \kappa^{3-D}}{(4 \pi)^{D / 2}} \int_{0}^{\ell} d \tau_{1} \int_{0}^{\ell} d \tau_{2} \dot{x}^{\mu}\left(\tau_{1}\right) \dot{x}_{\mu}\left(\tau_{2}\right) \int_{0}^{\infty} \frac{d \lambda}{\lambda^{D / 2}} e^{-\lambda m^{2}} e^{-\left(x\left(\tau_{1}\right)-x\left(\tau_{2}\right)\right)^{2} / 4 \lambda}
$$

where eq. (3.7) has been used for the integration over the momenta in $f_{3}\left(\tau_{1}, \tau_{2}\right)$. Next we write

$$
F_{3}(C, m)=\sum_{i=1}^{3} F_{3, \mathcal{D}_{i}}
$$

with the domains $\mathcal{D}_{i}$ as in eq. (3.5) and

$$
F_{3, \mathcal{D}_{i}}=\frac{2 m \kappa^{3-D}}{(4 \pi)^{D / 2}} \iint_{\mathcal{D}_{i}} d \tau_{1} d \tau_{2} \dot{x}^{\mu}\left(\tau_{1}\right) \dot{x}_{\mu}\left(\tau_{2}\right) \int_{0}^{\infty} \frac{d \lambda}{\lambda^{D / 2}} e^{-\lambda m^{2}} e^{-\left(x\left(\tau_{1}\right)-x\left(\tau_{2}\right)\right)^{2} / 4 \lambda} .
$$

Finally, we analyze each one the integrals $F_{3, \mathcal{D}_{i}}$.

We start with $F_{3, \mathcal{D}_{1}}$. Analogous arguments to those used for $F_{2, \mathcal{D}_{1}}$ give

$$
\begin{aligned}
F_{3, D_{1}} & =-\frac{2 m \kappa^{3-D}}{(4 \pi)^{D / 2}}\left\{\ell \int_{0}^{\delta} d t \int_{0}^{\infty} \frac{d \lambda}{\lambda^{D / 2}} e^{-\lambda m^{2}} e^{-t^{2} / 4 \lambda}\right. \\
& \left.+\int_{0}^{\ell} d \tau_{1} \sum_{j=2}^{\infty} \sum_{k=0}^{[j / 4]} \tilde{c}_{j k}\left(\tau_{1}\right) \int_{0}^{\delta} d t t^{j} \int_{0}^{\infty} \frac{d \lambda}{\lambda^{(D+2 k) / 2}} e^{-\lambda m^{2}} e^{-t^{2} / 4 \lambda}\right\},
\end{aligned}
$$

where $\tilde{c}_{j k}\left(\tau_{1}\right)$ are functions of $x^{\mu}\left(\tau_{1}\right)$ and its derivatives only. The first term on the right-hand side in eq. (3.17) is not well defined at $D=3$ and thus one has to 
keep $D$ arbitrary. Regarding the second term, the very same arguments as those used for eq. (3.11) show that it vanishes as $D \rightarrow 3, m \rightarrow \infty$. Hence, only the first term contributes to the double limit $D \rightarrow 3, m \rightarrow \infty$ :

$$
F_{3, \mathcal{D}_{1}}=-\frac{2 m \kappa^{3-D} \ell}{(4 \pi)^{D / 2}} \int_{0}^{\delta} d t \int_{0}^{\infty} \frac{d \lambda}{\lambda^{D / 2}} e^{-\lambda m^{2}} e^{-t^{2} / 4 \lambda}+\text { v.t. }
$$

Interchanging the order of integration over $t$ and $\lambda$ and making the changes $u=$ $m t$ and $\zeta=\lambda m^{2}$, we have

$$
F_{3, \mathcal{D}_{1}}=-\frac{m \ell}{4 \pi}\left(\frac{m^{2}}{4 \pi \kappa^{2}}\right)^{(D-3) / 2} \int_{0}^{\infty} \frac{d \zeta}{\zeta^{(D-1) / 2}} e^{-\zeta} \Phi\left(\frac{m \delta}{2 \sqrt{\zeta}}\right)
$$

where

$$
\Phi(x)=\frac{2}{\sqrt{\pi}} \int_{0}^{x} d u e^{-u^{2}}
$$

is the probability integral. Noting that

$$
\zeta^{(1-D) / 2}=\frac{2}{3-D} \frac{d}{d \zeta} \zeta^{(3-D) / 2}
$$

integrating by parts and expanding $\left(4 \pi \kappa^{2} m^{-2} \zeta\right)^{(3-D) / 2}$ in powers of $D-3$, we obtain

$$
\begin{aligned}
F_{3, \mathcal{D}_{1}} & =\frac{m \ell}{2 \pi} \frac{1}{D-3}+\frac{m \ell}{4 \pi} \ln \left(\frac{m^{2}}{4 \pi \kappa^{2}}\right) \\
& +\frac{m \ell}{4 \pi} \int_{0}^{\infty} d \zeta \ln \zeta \frac{d}{d \zeta}\left[e^{-\zeta} \Phi\left(\frac{m \delta}{2 \sqrt{\zeta}}\right)\right]+O(D-3) .
\end{aligned}
$$

The first term on the right-hand side gives a pole in $D-3$, whereas the second and third terms give finite non-vanishing contributions. It can be seen after some 
calculus that the large $m$ limit of the integral in the third term is given by

$$
m \int_{0}^{\infty} d \zeta \ln \zeta \frac{d}{d \zeta}\left[e^{-\zeta} \Phi\left(\frac{m \delta}{2 \sqrt{\zeta}}\right)\right]=m \gamma+\text { terms that vanish as } m \text { goes to } \infty
$$

where $\gamma$ is the Euler constant. We then conclude that

$$
F_{3, \mathcal{D}_{1}}=\frac{m}{2 \pi}\left[\frac{1}{D-3}+\frac{1}{2} \ln \left(\frac{m^{2}}{4 \pi \kappa^{2}}\right)+\frac{1}{2} \gamma\right] \ell+\text { v.t. }
$$

Using the same type of arguments it is not difficult to see, though a bit tedious, that

$$
\lim _{m \rightarrow \infty} \lim _{D \rightarrow 3} F_{3, \mathcal{D}_{2}}=0
$$

Concerning $F_{3, \mathcal{D}_{3}}$, it can be studied in the same way as $F_{2, \mathcal{D}_{3}}$. Indeed, for all $\left(\tau_{1}, \tau_{2}\right)$ in $\mathcal{D}_{3}$ we have $\left|x\left(\tau_{1}\right)-x\left(\tau_{2}\right)\right| \geq r_{0}>0$ and

$$
0 \leq\left|F_{3, \mathcal{D}_{3}}\right| \leq B_{0}(\delta) \kappa^{3-D}\left(\frac{m}{2 \pi r_{0}}\right)^{\frac{D}{2}-1} K_{\frac{D}{2}-1}\left(m r_{0}\right)
$$

with $B_{0}(\delta)$ a positive constant. So taking the limits $D \rightarrow 3$ and $m \rightarrow \infty$ and using eq. (3.14) we have

$$
\lim _{m \rightarrow \infty} \lim _{D \rightarrow 3} F_{3, \mathcal{D}_{3}}=0
$$

Putting together eqs. (3.16) and (3.18)-(3.20) we reach eq. (3.3). With this we complete the proof of eqs. (3.1)-(3.3), hence of eq. (2.13).

Acknowledgements: FRR was supported by FOM, The Netherlands. The authors are also grateful to CICyT, Spain for partial support. 


\section{References}

1. A. Schwartz, Commun. Math. Phys. 67 (1979) 1.

2. C. Tze, Int. J. Mod. Phys. A3 (1988) 1959.

E. Witten, Commun. Math. Phys. 121 (1989) 351.

3. G. Călugăreanu, Rev. Math. Pures Appl. 4 (1959) 5; Czech. Math. J. 11 (1961) 588.

W.F. Pohl, J. Math. Mech. 17 (1968)693.

J.H. White, Am. J. Math. 91 (1969) 693.

4. A.M. Polyakov, Mod. Phys. Lett. A3 (1988) 325.

5. F. Brock Fuller, Proc. Nat. Acad. Sci. USA 68 (1971) 815.

6. S. Deser, R. Jackiw and S. Templeton, Phys. Rev. Lett. 48 (1982) 975.

G. Dunne, R. Jackiw and C.A. Trugenberger, Phys. Rev. D41 (1990) 661.

7. G. Giavarini, C.P. Martin and F. Ruiz Ruiz, Nucl. Phys. B381 (1992) 222.

8. T.H. Hasson, A. Karlhede and M. Roček, Phys. Lett. B225 (1989) 92.

A. Coste and M. Makowka, Nucl. Phys. B342 (1990) 721.

9. K. Hepp, Thèorie de la renormalisation, Lectures Notes in Physics, vol. 2 (Springer, Berlin 1969).

10. A.M. Polyakov, Nucl. Phys. B164 (1979) 171.

V.S. Dotsenko and S.N. Vergeles, Nucl. Phys. B169 (1980) 527.

R.A. Brandt, F. Neri and M. Sato, Phys. Rev. D24 (1981) 879.

J.L. Gervais and A. Neveu, Nucl. Phys. B163 (1980) 189.

11. M. Asorey, F. Falceto and S. Carlip, Chern-Simons states and topologically massive gauge theories, University of California at Davis and University of Zaragoza preprints UCD-93-7 and DFTUZ 93.5 (hep-th/9304081).

12. H. Epstein and V. Glaser, Ann. Inst. Henri Poincaré XIX (1973) 211. 
13. J.C. Collins, Renormalization (Cambridge University Press, Cambridge 1987). 